\title{
Specificity of Reflex Adaptation for Task-Relevant Variability
}

\author{
David W. Franklin and Daniel M. Wolpert \\ Computational and Biological Learning Laboratory, Department of Engineering, University of Cambridge, Cambridge CB2 1PZ, United Kingdom
}

\begin{abstract}
The motor system responds to perturbations with reflexes, such as the vestibulo-ocular reflex or stretch reflex, whose gains adapt in response to novel and fixed changes in the environment, such as magnifying spectacles or standing on a tilting platform. Here we demonstrate a reflex response to shifts in the hand's visual location during reaching, which occurs before the onset of voluntary reaction time, and investigate how its magnitude depends on statistical properties of the environment. We examine the change in reflex response to two different distributions of visuomotor discrepancies, both of which have zero mean and equal variance across trials. Critically one distribution is task relevant and the other task irrelevant. The task-relevant discrepancies are maintained to the end of the movement, whereas the task-irrelevant discrepancies are transient such that no discrepancy exists at the end of the movement. The reflex magnitude was assessed using identical probe trials under both distributions. We find opposite directions of adaptation of the reflex response under these two distributions, with increased reflex magnitudes for task-relevant variability and decreased reflex magnitudes for taskirrelevant variability. This demonstrates modulation of reflex magnitudes in the absence of a fixed change in the environment, and shows that reflexes are sensitive to the statistics of tasks with modulation depending on whether the variability is task relevant or task irrelevant.
\end{abstract}

Key words: motor control; vision; reaching; reflexes; on-line control; adaptive control of feedback

\section{Introduction}

Previous studies examining reflex responses have found that the gain of a reflex response can be adapted by the CNS. Examples include, the vestibulo-ocular reflex under magnifying glasses (Robinson, 1976; Miles and Eighmy, 1980), the H-reflex between different locomotory activities (Capaday and Stein, 1986; Llewellyn et al., 1990), and reflexes during postural tasks (Nashner, 1976; Akazawa et al., 1983; Horak and Nashner, 1986; Doemges and Rack, 1992a,b), catching tasks (Lacquaniti and Maioli, 1987; Lacquaniti et al., 1991, 1992), unstable environments (De Serres and Milner, 1991; Fitzpatrick et al., 1994; Franklin et al., 2007), cyclical movements (Dufresne et al., 1980; Brown and Kukulka, 1993; Johnson et al., 1993; Pearson and Misiaszek, 2000; Zehr et al., 2003) or under different task instructions (Hammond, 1956; Evarts and Tanji, 1974; Barr et al., 1976; Crago et al., 1976; Rothwell et al., 1980; Pruszynski et al., 2008) or limb dynamics (Lacquaniti and Soechting, 1984, 1986a,b; Kurtzer et al., 2008). However, in all these cases the change in the reflex response is induced either by a fixed change in the environment, e.g., magnifying glasses, or by task requirements linked to different states of the body e.g., different stages of locomotion. Here we investigate whether a change in the reflex magnitude occurs in

\footnotetext{
Received Sept. 15, 2008; revised 0ct. 20, 2008; accepted Nov. 13, 2008.

This work was supported by the Wellcome Trust and the European project SENSOPAC IST-2005-028056. D.W.F. is supported by a fellowship from Natural Science and Engineering Research Council, Canada. We thank I. Howard and J. Ingram for their work in setting up the VBOT robotic interface used in this study. We thank S. Franklin for her assistance with the experiments.

Correspondence should be addressed to David W. Franklin, Department of Engineering, University of Cambridge, Trumpington Street, Cambridge CB2 1PZ, UK. E-mail: dwf25@cam.ac.uk. D0I:10.1523/JNEUROSCI.4406-08.2008

Copyright $\odot 2008$ Society for Neuroscience 0270-6474/08/2814165-11\$15.00/0
}

response to a varying environment in which the net disturbance is zero. To do this we examine a visuomotor reflex that allows precise control over the statistics of the disturbance.

Rapid motor responses to visual signals occur in response to the presentation of a visual stimulus (Corneil et al., 2004, 2008), shifts in the target location (Soechting and Lacquaniti, 1983), the entire background (Saijo et al., 2005) and in the representation of the hand position (Sarlegna et al., 2003; Saunders and Knill, 2003, $2004,2005)$. The visually induced corrective responses to target jumps occur relatively quickly $(150 \mathrm{~ms})$ after the representation of the hand or target moves and do not require subjects to consciously perceive these movements (Goodale et al., 1986; Prablanc and Martin, 1992). While this previous work has shown fast visually induced motor reflexes to movements of the target (Day and Lyon, 2000) or background (Saijo et al., 2005; Gomi et al., 2006), it has not previously been clear that responses to the visual movement of the hand location (visuomotor discrepancy) rely on an involuntary reflex response. Here, we show that this response is reflexive in nature and, moreover, we examine the change in the magnitude of the visuomotor reflex induced by a statistical distribution of discrepancies experienced over many trials. The mean visuomotor discrepancy was chosen to be zero with a fixed variance across trials. Therefore, this discrepancy added visual uncertainty about the location of the hand position. In one condition the visuomotor discrepancy was maintained until the end of the movement while in a second condition the discrepancy was transitory: the former represents task relevant variability because it requires a corrective response to reach the target while the latter is task irrelevant because no correction is necessary for task achievement. We examine the effect of the forms of variability on reflex adaptation. 


\section{Materials and Methods}

Ten subjects (six male and four female) with no reported neurological disorders participated in the main study (mean age: $26 \pm 5$ years). A second group of 10 subjects (eight male and two female) (mean age: $31 \pm 5$ years), one of whom also participated in the main study, were recruited to participate in a control experiment. All subjects were right-handed according to the Edinburgh handedness inventory (Oldfield, 1971). Subjects gave informed consent and the experiments were approved by the institutional ethics committee.

Experimental setup. Movements investigated in this study were right-handed forward reaching movements in the horizontal plane at $\sim 10$ $\mathrm{cm}$ below the subjects' shoulder level. The forearm was supported against gravity with an air sled. The handle of the robotic manipulandum (vBOT) used to generate the environmental dynamics was grasped by the subject (Fig. 1A). Position and force data were sampled at $1 \mathrm{kHz}$. Endpoint forces at the handle were measured using an ATI Nano 25 six-axis force-torque transducer (ATI Industrial Automation). Visual feedback was provided using a computer monitor mounted above the vBOT and projected veridically to the subject via a mirror. This virtual reality system covers the manipulandum, arm and hand of the subject preventing any visual information of their location. The delay of the computer monitor for presentation of visual feedback was determined to be $16 \mathrm{~ms}$. Results in this study are presented relative to the onset of the actual perturbation time. This means that the monitor delay has been taken into account such that delay times are relative to the time at which the visual signal was actually presented.

Movements were made from a $1.0 \mathrm{~cm}$ diameter start circle centered $28.0 \mathrm{~cm}$ in front of the subject to a $2.0 \mathrm{~cm}$ diameter target circle centered $25 \mathrm{~cm}$ in front of the start circle. The subjects' arm was hidden from view by the virtual reality visual system on which the start and target circles, as well as a $0.6 \mathrm{~cm}$ diameter cursor used to track instantaneous hand position, were projected. Subjects were instructed that they were required to perform successful movements to complete the experiment. Successful movements were defined as those which entered the target without overshooting and with movement durations in the range $700 \pm 75 \mathrm{~ms}$. When subjects performed successful movements, a counter increased, and subjects were instructed that they needed to keep making successful movements until the counter reached a certain number (200 movements in each phase of the experiment). When subjects performed unsuccessful movements they were provided with feedback as to why the movement was not considered successful ("too fast," "too slow," "overshot target"). Trials were self paced; subjects initiated a trial by moving the hand cursor into the start circle and holding it within the target for $450 \mathrm{~ms}$. A beep then indicated that the subjects could begin the movement to the target. The duration of the movement was determined from the time that the subjects exited the start target until the time that subject's entered the final target.

Electromyography. Surface electromyography (EMG) was recorded from two monoarticular shoulder muscles: pectoralis major and poste-
A

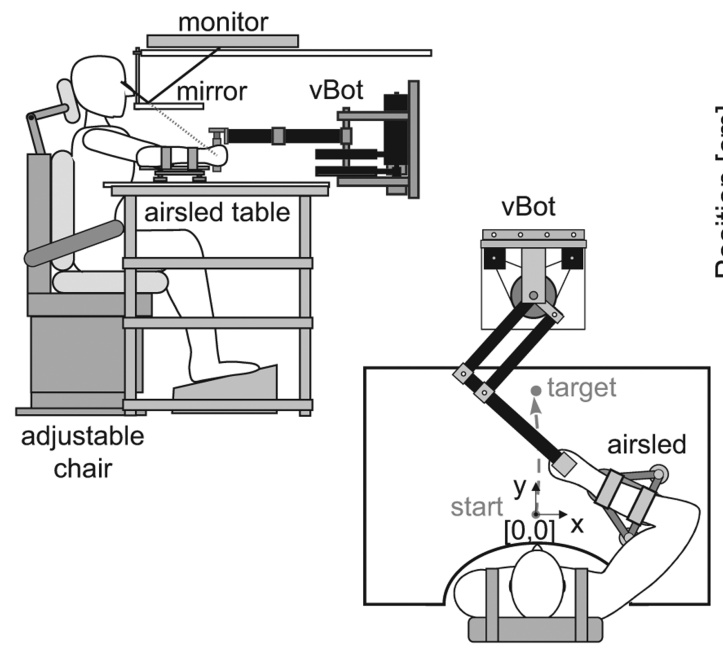

C

React in Same Direction D as Perturbation
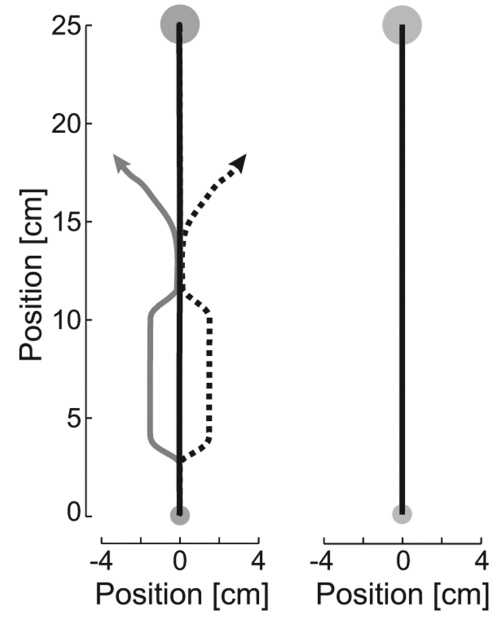

Position [cm]
B Visual Perturbations

(Probe Trials)

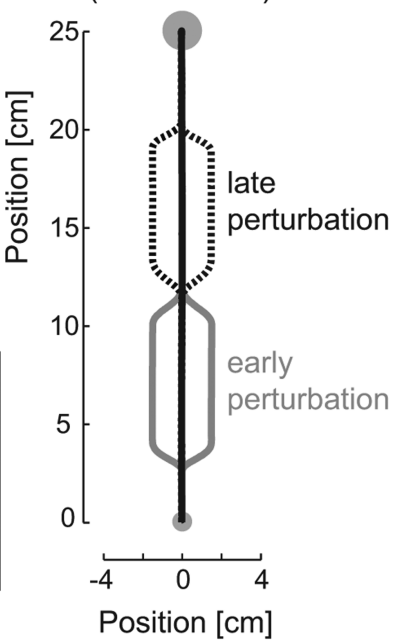

Figure 1. The experimental setup. $\boldsymbol{A}$, The subject grasps the robotic vBOT while seated. Visual feedback is presented veridically using a top mounted computer screen viewed through a mirror. The subject's forearm is fixed to and supported by an airsled. $\boldsymbol{B}$, The visual perturbations (probe trials) used to examine the magnitude of the visually induced motor response. While the hand was mechanically constrained to a straight line trajectory to the target, the visual cursor representing the hand to the subject was smoothly displaced using a ramp, hold and ramp back to the actual hand position. $C$, Voluntary response experiment. On random trials the hand cursor is perturbed by the probe trials while the hand is constrained by a mechanical channel. Subjects are instructed to move their hand as quickly as possible in the same direction as the perturbation. $\boldsymbol{D}$, Visuomotor discrepancy experiment. The visual cursor representing the subject's hand was manipulated in one of three ways in the latter half of the movements. In the normal condition (left) the cursor reproduced the hand trajectory exactly. In the task-relevant condition, the visual cursor smoothly moved away from the hand trajectory to one of seven amplitudes and remained as this point for the rest of the movement. In the task-irrelevant condition, the visual cursor smoothly moved away from the hand trajectory exactly as it did in the task-relevant condition but then returned smoothly such that it agreed with the physical hand position at the end of the movement. The double-sized task-irrelevant condition (right) was the same as the task-irrelevant condition but with amplitudes of twice the size.

rior deltoid; two biarticular muscles: biceps brachii and long head of the triceps; and two monoarticular elbow muscles: brachioradialis, and lateral head of the triceps. EMG was recorded using the Delsys Bagnoli (DE-2.1 Single Differential Electrodes) electromyography system. The electrode locations were chosen to maximize the signal from a particular muscle while avoiding cross talk from other muscles. The skin was cleansed with alcohol and prepared by rubbing an abrasive gel into the skin. This was removed with a dry cloth and the gelled electrodes were secured to the skin using double-sided tape. The EMG signals were ana$\log$ bandpass filtered between 20 and $450 \mathrm{~Hz}$ (in the Delsys Bagnoli EMG 
system) and then sampled at $2.0 \mathrm{kHz}$. The muscle activity of one subject was not included in the analysis due to the presence of noise in the signal

Probe trials. Visually induced motor responses were examined in all the experiments by introducing a ramp and hold perturbations of the visual system (Fig. $1 B$ ). In the middle of the movements to the target, the cursor representing the hand position was smoothly ramped away from the current hand position $>50 \mathrm{~ms}$, held at a distance of $1.5 \mathrm{~cm}$ for $130 \mathrm{~ms}$ and then smoothly returned to the actual hand position during the movement. The entire visual perturbation lasted for $230 \mathrm{~ms}$. During this trial, the hand was physically constrained to the straight path between the start and final targets (mechanical channel trial generated by the vBOT). By constraining the physical hand location using the channel, no change in the arm configuration will occur, potentially producing stretch reflex responses or other physically induced motor responses that could contribute to the force or muscle activity. Second, any force produced in response to the visual perturbation can be measured against the channel wall using the force sensor. The other main property of this visual perturbation is that it returns to the actual hand trajectory. This means that the subjects do not need to respond to the visual perturbation to produce a successful movement to the target. This is in contrast to previous studies examining the responses to visual perturbations of the hand location, all of which required subjects to compensate for the visual disturbance (Sarlegna et al., 2003, 2004; Saunders and Knill, 2003, 2004, 2005). These visual perturbations were applied at two different points during the movement (early: $10 \%$ of the movement distance; late: $45 \%$ of the movement distance) either to the left or right. For comparison a zero perturbation trial was also included in which the hand was held to a straight line trajectory to the target but the visual cursor remained at the hand position throughout the trial. The five perturbation trials were randomly applied during movements in a blocked manner such that each of the five perturbations was applied within a block of trials. All references within this study to a visual perturbation refer to these fast changes in visual feedback that occur on probe trials.

Experiment 1: timing of voluntary response. A shift in the visual location of the hand produces a corrective motor response (Saunders and Knill, 2003, 2004). However, previous experiments have not confirmed whether this motor response is voluntary or involuntary in nature. This experiment was performed to determine the fastest time of voluntary response to a shift in the visual location of the hand and whether the motor response occurred before this time or not. In particular, we used a technique similar to that of Day and Lyon (2000) where subjects were asked to move in the same direction as a visual perturbation as quickly as possible. Because the motor response to the visual perturbation produces a force opposite to the perturbation direction, the main question is whether this response would still be present under this condition. If this response was voluntary in nature, we would expect that it would not longer be present. However, if the response was involuntary in nature then we would expect it to occur before the timing of the requested voluntary response and in the direction opposite to the voluntary response. Subjects were instructed to make reaching movements to the target such that a total of 200 successful movements were performed. On a random $40 \%$ of trials, a visual perturbation (probe trial) was applied in one of the two directions (left or right) at one of the two timings (early or late) as described previously under the heading "probe trials." Subjects were instructed to look for these visual perturbations and respond to them by producing a movement as quickly as possible in the same direction as the visual perturbation (Fig. 1C). If a visual perturbation was produced then subjects were automatically credited with a successful trial.

Experiment 2: visuomotor discrepancy. This experiment was performed to examine whether the magnitude of the visually induced motor response can be controlled by the CNS and whether it is sensitive to the statistics of the physical world. Subjects performed reaching movements in several environments with various degrees of visuomotor discrepancies. Within each environment, the visually induced motor response magnitude was assessed on random trials by the use of probe trials (described in detail above). As the same probe trials were used in each of the environments, any changes in the magnitude of the motor response could be attributed to the influence of the surrounding trials. On all trials except for those containing probe trials, the hand was free to move within the plane. On the probe trials, the physical location of the hand was laterally constrained to move within the mechanical channel to the target as described previously.

As the probe trials were presented on random trials throughout the experiments in all of the fields, the time course of any adaptation from one environment to the next, or throughout the trials within an environment, could be assessed. In particular, subjects always started making movements in the normal environment (pre-normal), then either in the task-relevant or task-irrelevant environments, and finally back in the normal environment (post-normal). In this way any effects of the environments containing the visual discrepancies could be assessed against both the pre-exposure and post-exposure results in the normal environment.

Visual environments. Subjects made movements in one of three visual environments: normal, task-relevant and task-irrelevant (Fig. $1 D$ ). In the normal condition there was no discrepancy between the visual path of the hand and the actual physical path of the hand. Subjects made straight movements to the target directly in front of them. In the task-relevant condition, at a point in the trajectory that was $40 \%$ of the distance to the target $(10 \mathrm{~cm}$ from the start), the visual cursor representing the hand position underwent a smooth (minimum jerk) movement laterally to the movement direction to a distance from the set $[-3,-2,-1,0,1,2,3] \mathrm{cm}$ in $7.5 \mathrm{~cm}$ of the forward movement distance and remained at this location. Subjects were required to determine the appropriate response to bring the hand cursor back in to the target and be credited with a successful trial. In this condition, while the lateral change in the visual location of the hand position produces a visuomotor discrepancy, the visual signal provides reliable information about the amount of compensation that the subjects will need to produce to successfully complete the movement. In the task-irrelevant condition, $40 \%$ of the distance to the final target $(10 \mathrm{~cm}$ from the start), the visual cursor representing the hand position underwent a smooth movement laterally (minimum jerk) to a distance from the set $[-3,-2,-1,0,1,2,3] \mathrm{cm}$ in $7.5 \mathrm{~cm}$ of forward movement and then returned to the actual hand position in the next 7.5 $\mathrm{cm}$ of forward movement in the same manner such that at the final target the hand position and cursor position were matched. While this condition initially produces an identical visuomotor discrepancy to the taskrelevant condition, the visual signal does not provide reliable information about the location of the hand at the end of the trial. Subjects returned their own hand to the start position, however no visual feedback of the hand position was provided until the subject's hand was within the final $5 \mathrm{~cm}$ of the start position. In such a manner, any mismatch between the subject's hand position and the target position due to the visuomotor discrepancy that might exist at the end of the movements was not obvious to the subjects.

Assessment of reflex magnitude. In all three environments the five different visual perturbation trials or probe trials (Fig. $1 B$ ) (as described in the section entitled probe trials) were presented on one third of the trials (5 probe trials within every 15 trials) to assess the reflex response. The first possible probe trial in a new environment occurred on the fifth trial such that subjects always experienced at least four trials in the environment before the first measurement of the reflex response. While lateral movement in the random probe trials was constrained by the mechanical channel, the subjects were free to move in any manner during all of the other trials.

Visual discrepancies and visual perturbations. These two terms are used within this manuscript to refer to two distinct visual manipulations. Visual discrepancies refer to the slow and smooth minimum jerk changes in the visual location of the hand which are applied to create the taskrelevant and task-irrelevant environments (Fig. $1 D$ ). In contrast, the term visual perturbation refers to the fast $230 \mathrm{~ms}$ shift in the visual feedback of the hand location which occurs on the probe trials (Fig. $1 B$ ) contained within all environments. All measurements of the reflex magnitude were made using these visual perturbations on the probe trials.

Protocol. To examine the effects of each of the two changed conditions (task-relevant or task-irrelevant) the responses were contrasted with the normal condition to get an appropriate baseline for both conditions. Subjects were randomly split into two groups. The first group experi- 
enced the task-relevant condition on day 1 and the task-irrelevant condition on day 2 , whereas the second group experienced the conditions in the opposite order. On each day subjects first performed 200 successful movements in the normal condition (prenormal condition). After a short break the subjects performed 200 successful movements in either the task-relevant condition or task-irrelevant condition, followed again by a short break and 200 successful movements in the normal condition (postnormal condition).

Control condition. The visuomotor discrepancies (task-relevant and task-irrelevant) were matched in their amplitudes. However, the integrated signal size (over time or distance) would be smaller in the taskirrelevant environment. To demonstrate that any difference in the responses was not due to simply to differences in the integrated size, 10 subjects were recruited to perform a control condition in a taskirrelevant environment with amplitudes of double the original size (Fig. $1 D)$. The peak amplitudes for the visual discrepancies came from the set $[-6,-4,-2,0,2,4,6] \mathrm{cm}$. Subjects first made movements in the null environment, followed by movements in the double-sized taskirrelevant environment after a short break. Finally after a second break, subjects again performed movements in the null environment. In contrast to the first set of experiments, subjects were required to make 300 successful movements in each phase of the experiment. All other parameters of the experimental design were kept constant.

Analysis. Analysis of the experimental data were performed using Matlab R14. EMG data were highpass filtered at $30 \mathrm{~Hz}$ (fifth order Butterworth) to remove any movement artifact or offset. Individual trials were aligned on visual perturbation onset and averaged across repetitions. The response to the right visual perturbation was subtracted from the response to the left perturbation to provide a single estimate of the motor response to the visual perturbation. ANOVAs were examined in SPSS 16.0 using the general linear model. If a significant main effect was found, Tukey's honest significant difference (HSD) post hoc test was used to examine differences. Statistical significance was considered at the $p<$ 0.05 level for all statistical tests.

To examine how quickly subjects were able to adapt to the visual discrepancies, two measures were examined. The first was the time it took subjects to place the cursor into the target measured from the onset of the discrepancy (or from the time at which the hand was at $10 \mathrm{~cm}$ from the start position for the normal condition). The second was the maximum perpendicular distance to the straight line joining the start and end target after the visual discrepancy onset. This was a measure of the amount to which subjects responded to the sudden shift in the visual cursor.

The time course of the force response during the visuomotor discrepancy experiment was examined for each day of experiment 2 . Each of the five perturbations or probe trials was applied randomly within each block of 15 trials during the experiments. Therefore an estimate of the force response magnitude can be made for each block of 15 trials. The speed at which these visually induced motor response magnitudes adapt as subjects proceeded from the normal environment (prenormal) to either the task-relevant or task-irrelevant environment and then again back to the normal environment (postnormal) can then be determined. The difference in the force response between the left and right visual perturbations was determined for each block of 15 trials where one of each type of visual perturbation was applied. These were determined for each subject separately for each block of trials. For the main study, the first 14 blocks (the first 215 trials), where data for all subjects existed, was used. For the control condition, the first 20 blocks ( 305 trials) were used. To examine whether the magnitude of the response was affected by learning within a condition, and to see whether the response was immediately changed as subjects went from one condition to another, the force response on the first block and the last three blocks for each condition within a single day were examined (except for the first block in the prenormal condition) with an ANOVA. If a significant main effect of block (five levels) was found, a Tukey's HSD post hoc test was used to examine whether significant differences existed between different blocks.

\section{Results}

Subjects performed reaching movements while holding the handle of a robotic manipulandum (Fig. $1 A$ ). On random trials dur- ing normal reaching movements subjects were presented with a visual perturbation of the hand cursor while the hand itself was mechanically constrained to move within a channel to the target. These perturbations occurred either to the left or right of the hand trajectory early or late in the movement (Fig. $1 B$ ). On these trials the response to the visual perturbation (Fig. $2 A$ ) was measured by the force exerted against the channel wall (Fig. $2 B$ ). A rightward visual perturbation of the hand led to subjects generating a force into the leftward wall of the channel. This response is oriented in the appropriate direction to compensate for the movement of the hand had it been physically moved. The opposite response was seen in response to a leftwards visual perturbation. To quantify these responses, the differences between the force response to leftward and rightward visual perturbations were calculated, showing a clear motor response to the visuomotor perturbation (Fig. 2C).

To examine the onset time of this response, the difference in the response (force and EMG) between the right and left visual perturbations was determined for all trials. By averaging across all trials, the onset time for each subject was estimated. The onset time (averaged across early and late perturbations) of the response $\pm \mathrm{SD}$ was $151.5 \pm 8.0 \mathrm{~ms}$ in the force (Fig. $3 A$ ), $108.3 \pm$ $12.8 \mathrm{~ms}$ in the pectoralis major (Fig. $3 B$ ) and $112.7 \pm 13.7 \mathrm{~ms}$ in the posterior deltoid (Fig. 3C).

\section{Experiment 1: timing of voluntary response}

To examine whether this response is reflex or voluntary in nature we performed a voluntary response experiment. Subjects were asked to make reaching movements with visual perturbations interspersed randomly on $40 \%$ of the trials. Subjects were instructed that if they saw a visual perturbation they should react as fast as possible by producing a movement in the same direction as the perturbation (Fig. $1 C$ ). However, before the voluntary response, subjects produced an initial force in the opposite direction (Fig. $4 A, B$ ). This is the direction which would act to restore the hand back toward the original trajectory. Similar responses were seen in both the pectoralis major (Fig. 4C) and Posterior deltoid (Fig. 4D) muscles with an earlier onset. The time of the voluntary response was estimated for each subject. The mean response times $( \pm \mathrm{SD})$ were $324 \pm 76 \mathrm{~ms}$ from the onset of the visual perturbation in the force and $232 \pm 38 \mathrm{~ms}$ from the onset of the visual perturbation in the EMG. Across all subjects no voluntary response was seen before $185 \mathrm{~ms}$ in the EMG and 232 $\mathrm{ms}$ in the force from the onset of the visual perturbation.

To confirm that the early response was consistently seen across all subjects, the mean response was estimated between 130 and $230 \mathrm{~ms}$ (force) and between 90 and $180 \mathrm{~ms}$ (EMG) after the onset of the perturbation. These responses were compared with the baseline measure estimated over another $100 \mathrm{~ms}$ interval occurring before any response in the signals $(-50-50 \mathrm{~ms})$ using a one tailed paired $t$ test. For both the early and late visual perturbations there was a significant force response occurring before the voluntary response and in the opposite direction (early: $t_{(9)}=$ $3.8, p=0.004$; late: $t_{(9)}=7.0, p<0.001$ ). Similar changes in the muscle activity relative to baseline were also seen in the pectoralis major (early: $t_{(8)}=2.0, p=0.041$; late: $t_{(8)}=2.2, p=0.031$ ) and the posterior deltoid (early: $t_{(8)}=3.9, p=0.002$; late: $t_{(8)}=3.9$, $p=0.002)$.

\section{Experiment 2: effect of visual environment on visually induced motor response}

To examine if the magnitude of the visually induced motor response could be modulated by the statistics of the task, subjects 
A
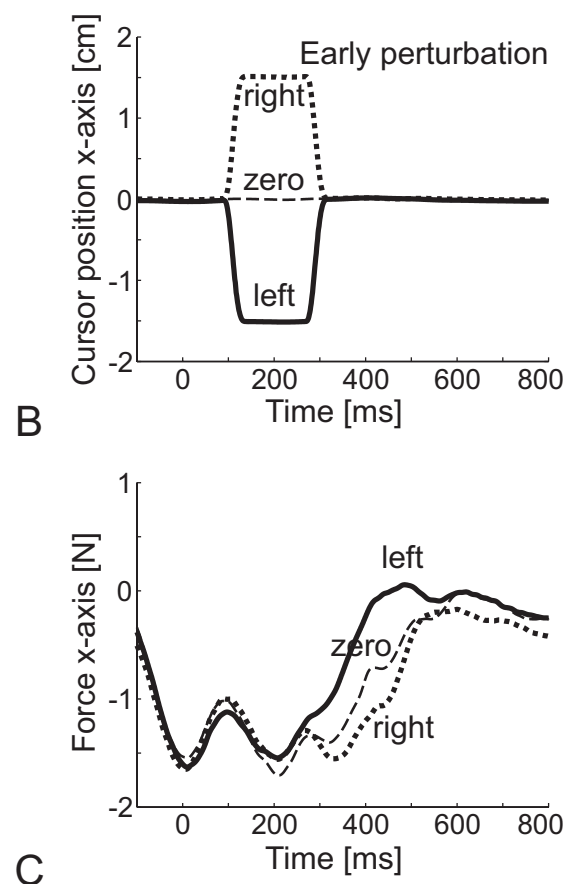

C

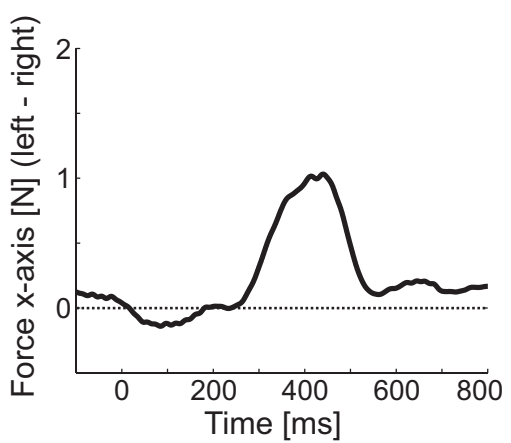

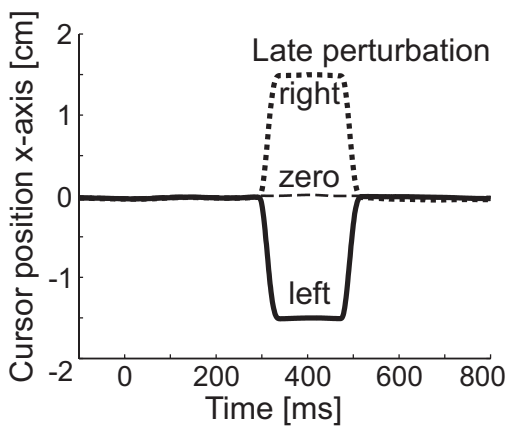
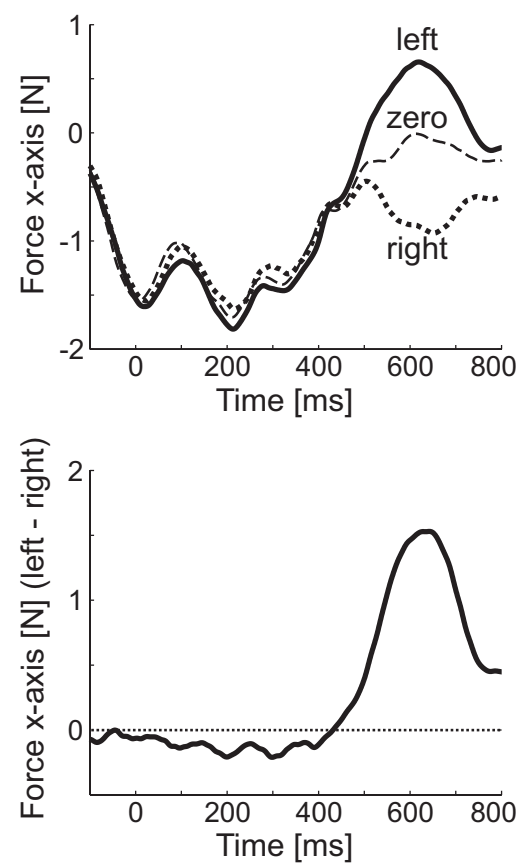

Figure 2. A motor response at the hand induced by a visual shift in the hand position during movement. Responses are shown for a single representative subject during movements in the normal condition. $\boldsymbol{A}$, The visual hand cursor perturbation in the $x$-axis is shown for both the early (left) and late (right) perturbations. $\boldsymbol{B}$, The force traces recorded against the channel wall with the force sensor. Forces in response to each visual perturbation are directly opposite to the visual perturbation. $\boldsymbol{C}$, The difference between the left and the right perturbations is determined to quantify the overall response to the visual perturbations.

performed movements in two different distributions of visuomotor discrepancies (Fig. 1D). In one condition subjects experienced a task-relevant set of discrepancies (Fig. $1 D$, middle) in which a visuomotor discrepancy of random size was introduced midway through the movement and maintained until the end of the movement. This required subjects to adapt their trajectory to reach the target. In a second condition (Fig. $1 D$, right), a visuomotor discrepancy of random size was introduced midway through the movement. This discrepancy was transitory; therefore subjects could ignore the discrepancy and still reach the target. Both before and after these discrepancies, subjects made reaching movements under veridical visual feedback where their hand path was roughly straight (Fig. 5A, $B$, left).

In the task-relevant environment, subjects compensated for the shifts in visual trajectory by moving the hand to the left or right so that the cursor was correctly positioned within the target (Fig. 5A, $B$, middle). In the task-irrelevant condition, subjects learned to ignore the visual discrepancy and make smooth straight movements to the target similar to those performed in the normal condition (Fig. $5 A, B$, right). To examine how quickly subjects were able to adapt to the visual discrepancies, two measures were examined. The first was the time, from the onset of the discrepancy, that it took subjects to reach the target. The second was the maximum perpendicular distance that the hand deviated from the line joining starting and target location. In the taskrelevant condition subjects quickly responded to the visual discrepancy (Fig. $5 C, E)$ thereby reducing the time taken to move the cursor into the target. In the task-irrelevant condition, both measures demonstrate that subjects initially responded to the shift in the cursor (in the first block of six movements) but quickly learned to ignore the shift in the cursor trajectory and move directly to the target (Fig. 5D,F).

While subjects performed reaching movements, probe trials containing a quick visual perturbation were randomly interspersed to elicit the visually induced motor response and determine whether the magnitude of the response varied depending on the visual environment. The difference in the force response to these visual perturbations to the left and right of the trajectory was calculated for both the early and late perturbations (Fig. 6). To examine whether the size of the reflex response in the task-relevant environment (Fig. 6A) had been modified, the mean force response over the early interval before the possible onset of voluntary activity (180-230 ms from the onset of the visual perturbation) was calculated for all conditions (Fig. 6D). A one-way repeated measure ANOVA was performed with 6 levels (3 visual environments (pre normal, task-relevant, post normal) $\times 2$ perturbation times (early, late)) was performed to examine if different responses were produced in the different environments. After a significant main effect $\left(F_{(5,49.9)}=14.405 ; p<0.001\right)$ was found, post hoc tests (Tukey's HSD) were used to examine differences between the visual environments and perturbation times. For the early perturbation, the force response in the task-relevant visual environment was larger than the responses in either the pre (normal) condition $(p<0.001)$ or the post (normal) condition $(p<$ 0.001 ). However, the response in the pre and post conditions were not significantly different from each other $(p=0.97)$. The early perturbation occurred before the onset of the visual discrepancies in the modified visual environments; however the response in the task-relevant environment was still enhanced with respect to the preceding normal condition. A similar response was seen during the late perturbation. The task-relevant condition was found to have a significantly greater response than the pre $(p<0.001)$ or post $(p<0.001)$ visual conditions which were not significantly different from each other $(p=0.98)$. The post hoc tests were also used to contrast the force response to the early and late perturbations for each visual environment. The response to the late perturbation was significantly larger than that to the 
early perturbation in the task-relevant environment $(p<0.001)$, but no differences in the responses between the early and late perturbations were found in either the pre ( $p=0.97)$ or post $(p=0.98)$ environments. This demonstrates that the reflex response to the visual perturbations was modified with spatial specificity such that the response during the latter half of the movement, where the task-relevant discrepancies were present, was larger than that in the first half of the movement.

In contrast to the task-relevant condition, the force response during the taskirrelevant condition did not increase relative to the normal condition responses (Fig. 6B). The mean response over an early interval before any voluntary response (180-230 ms from the onset of the visual perturbation) was examined using a oneway repeated measure ANOVA with 6 levels (3 visual environments (pre normal, task irrelevant, post normal) $\times 2$ perturbation times (early, late)) (Fig. 6E). After a significant main effect $\left(F_{(5,46.9)}=4.671\right.$; $p=0.002$ ), post hoc tests (Tukey's HSD) were used to examine differences between the visual environments and perturbation times. The only difference in the reflex responses between any of the three visual environments in the early perturbations was a significant decrease between the pre and post normal environments $(p=0.01)$. However, for the late perturbation, no significant differences were found for any of the three environments $(p>0.53)$. There were also no significant differences between the reflex responses to the early and late perturbations in the pre $(p=0.76)$, task-irrelevant $(p=0.055)$, or post $(p=$ $0.055)$ environments.

The same analysis was also performed for the responses in the double sized task irrelevant condition (Fig. 6C,F). After a significant main effect $\left(F_{(5,45.2)}=11.155 ; p<0.001\right)$, post hoc tests (Tukey's HSD) were used to examine differences between the visual environments and perturbation times. The force response in the double sized task-irrelevant visual environment was smaller than the responses in either the pre (normal) condition or the post (normal) conditions for both the early $(p<0.001)$ and late perturbations $(p<0.001)$. Similarly, there was a significant decrease between the pre and post normal conditions for both the early $(p=0.008)$ and late perturbations $(p=0.008)$. The post hoc tests were also used to contrast the force response to the early and late perturbations for each visual environment. The response to the late perturbation was significantly larger than that to the early perturbation in the pre (normal) environment $(p=0.048)$ and post normal environment $(p=0.048)$, but no differences in the response between the early and late perturbations were found in the double-sized task-irrelevant environment ( $p=0.37$ ). Overall, these results indicate that the involuntary response to the visual perturbation was suppressed during movements in the task-irrelevant condition when the visual discrepancies were large enough.

Finally, we examined the time course of the changes in the early perturbation
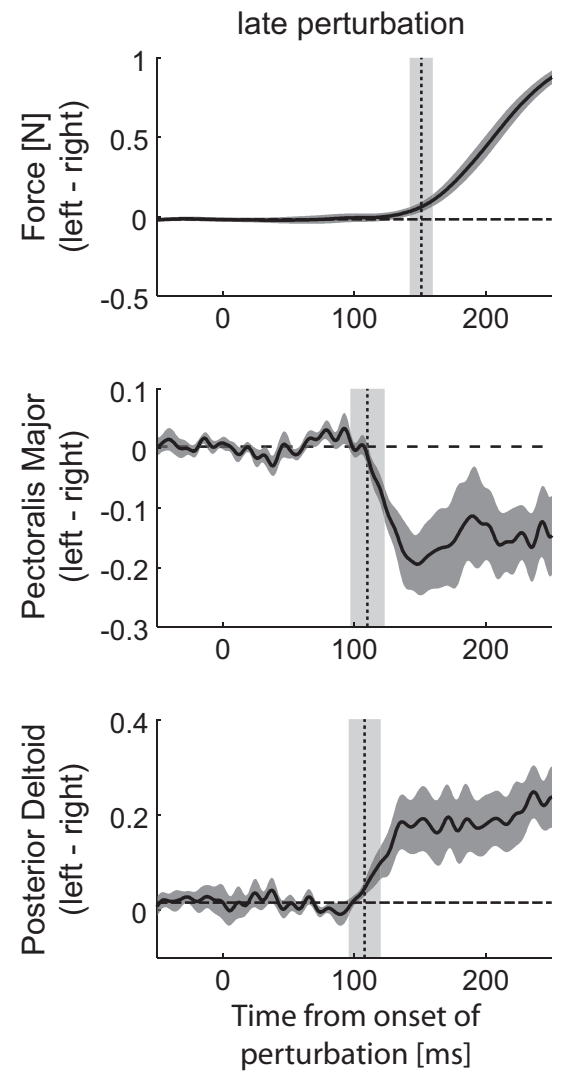

perturbation [ms]
perom onset of

perturbation [ms]

Figure 3. Onset of the involuntary visually induced motor response. $\boldsymbol{A}$, The mean \pm SEM (dark shaded region) of the force dotted vertical line indicates the mean \pm SD (light shaded region) onset of the force response as determined from each subject the perturbation. $\boldsymbol{B}$, The mean \pm SEM of the difference between the left and right visual perturbations in the pectoralis major muscle. $\boldsymbol{C}$, The mean \pm SEM of the difference between the left and right visual perturbations in the posterior deltoid muscle.

response. The mean force response across all subjects was calculated block by block throughout the experiment. This was performed either for the first 14 blocks of perturbation trials in the main study or for the first 20 blocks in the control condition (double-sized task-irrelevant condition) (Fig. 7) where data existed for all subjects. For each of the three experimental days (task-relevant, task-irrelevant, or double-sized task irrelevant), we compared the size of the force responses in the first and last three blocks (12th, 13th, and 14th blocks) of each of the three conditions (with the exception of the first block in the initial prenormal condition). These were contrasted using a repeated measures ANOVA with a main factor of block (five levels) for the response before the onset of voluntary reaction.

Within the first block of perturbations in the task-relevant condition, the force response increased, relative to the level in the pre normal condition, and remained at a high level throughout the entire condition. When subjects returned back to the normal (post) condition, the force response in the first block of trials was similar to the level in the task-relevant condition but gradually returned to baseline levels. After a significant main effect $\left(F_{(4,95)}\right.$ $=18.255 ; p<0.001)$, Tukey's HSD post hoc test indicated two homogeneous groups. The first contained the final blocks in the pre and post normal conditions which were not significantly different from each other $(p=0.981)$. The second contained the first and final three blocks in the task-relevant condition as well as the first block in the final normal condition (post). These three 
A

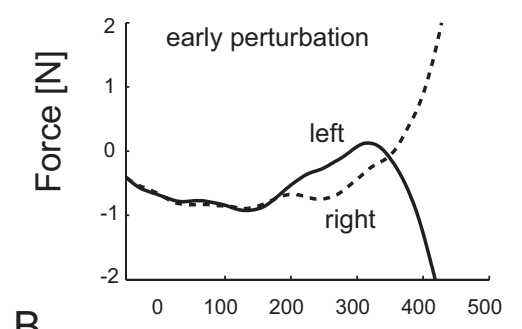

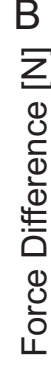

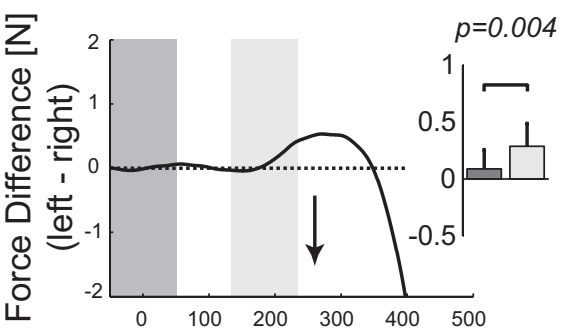

C
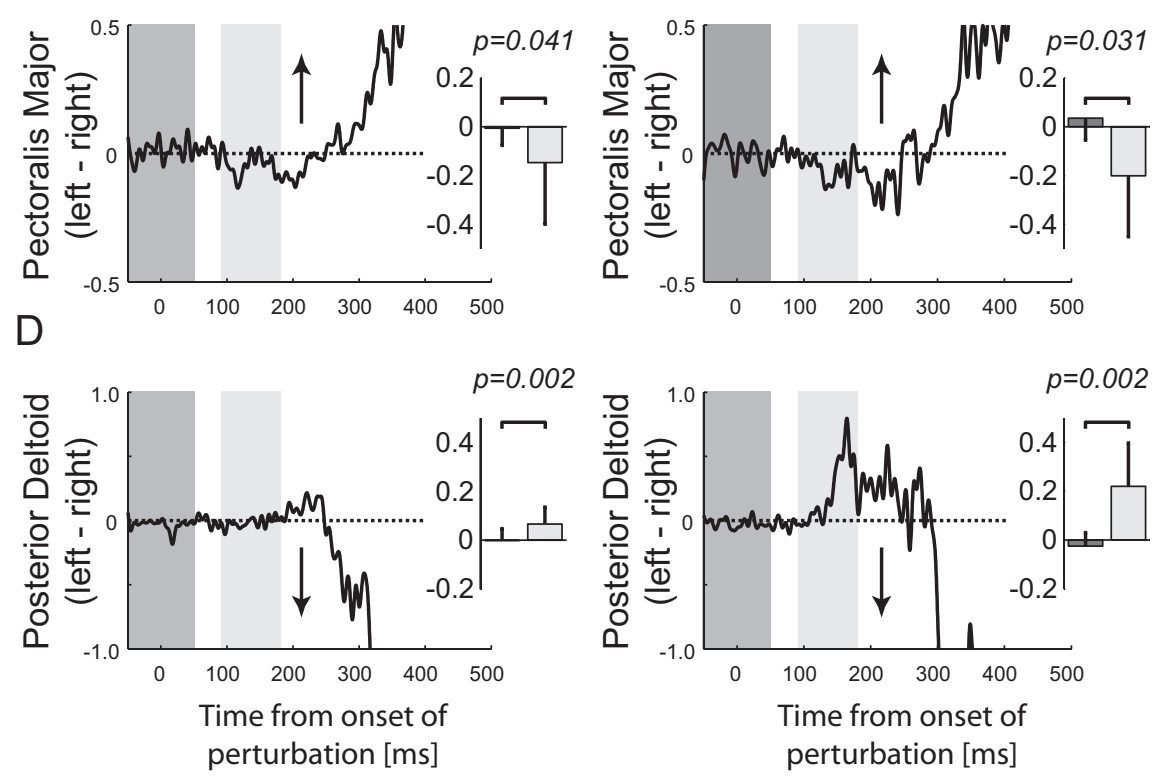

Figure 4. Timing of voluntary responses to visual perturbations. Responses to the early perturbation (left) and the late perturbation (right) are shown for a representative subject (traces) and across all subjects (bars graphs). Arrows indicate the expected direction of response based on the voluntary instruction. $\boldsymbol{A}$, The force responses against the channel wall plotted as a function of the time relative to the onset of the visual perturbation. Before the voluntary response at $\sim 350 \mathrm{~ms}$, the opposite response, producing a force in opposition to the visual perturbation is seen. $\boldsymbol{B}$, The difference between the left and right force traces. The response, determined over a $100 \mathrm{~ms}$ interval from $130 \mathrm{~ms}$ to $230 \mathrm{~ms}$ after the onset of the perturbation, was compared with the baseline, determined using a $100 \mathrm{~ms}$ interval from -50 to $50 \mathrm{~ms}$ as shown by the shaded regions. The mean response $\pm S D$ (error bars) across all subjects are shown to the right in the bar graph. The $p$ values for any significant effects using a one tailed $t$ test are indicated. $\boldsymbol{C}$, The difference in the activity of the pectoralis major muscle between the visual perturbations to the left and right. Mean responses compared the response in the interval $90-180 \mathrm{~ms}$ to the baseline interval from -50 to $50 \mathrm{~ms}$. The averaged rectified EMG was low-pass filtered at $100 \mathrm{~Hz}$ (fifth order Butterworth) for display purposes. $\boldsymbol{D}$, The difference in the posterior deltoid muscle between the visual perturbations to the left and the right.

blocks were not significantly different from each other $(p>$ 0.995 ) but significantly different from the other two blocks ( $p<$ 0.001). These results indicate two interesting findings. First, that on the first block of trials within the task-relevant condition, subjects had already adapted the response and no further learning effects are seen. Second, that when subjects were moved back in to the normal condition, the response in the first block of trials was not significantly different from that within the task-relevant con- dition. The reduction in the response occurs slower than the increase in the response.

In the task-irrelevant condition there was no change in the response to the visual perturbation (Fig. 7B). Instead, throughout the entire experiment, the force response appeared to gradually decrease. We compared the size of the force responses in the first and final three blocks (12th, 13th, and 14th blocks) of the conditions using an ANOVA with a main factor of block (five levels). We found no significant main effect of block $\left(F_{(4,92)}=0.779 ; p=0.54\right)$ indicating that there were no significant changes across the conditions.

When subjects made movements in the double sized task-irrelevant environment, the response to the visual perturbation gradually decreased (Fig. 7C). Upon the removal of the visual discrepancy, when subjects moved again in the (post) normal condition, the force response gradually increases. We compared the size of the force responses in the first and final three blocks $\left(12^{\text {th }}, 13^{\text {th }}\right.$, and $14^{\text {th }}$ blocks $)$ of the conditions using an ANOVA with a main factor of block (5 levels). After a significant main effect was found $\left(F_{(4,96)}=5.197 ; p=\right.$ 0.001 ), Tukey's HSD post hoc test indicated two significantly different groups. The first contained the final blocks in the pre and post normal conditions, which were not significantly different from each other ( $p=0.61$ ). The second contained the final three blocks in the double-sized taskirrelevant condition, which was significantly different from both the final blocks in the pre normal condition ( $p=0.044)$ and from the final blocks in the post normal condition $(p<0.001)$. These results illustrate that in the large amplitude taskirrelevant conditions the force response to the visual perturbations is gradually suppressed. However when the visual discrepancies are removed, the force response gradually increases back to the original levels.

\section{Discussion}

The results of this study provide clear evidence that the brain responds to shifts in the visual location of the hand during movement by producing a reflexive motor response to restore the hand back toward the original trajectory. This reflexive response occurs with a $110 \mathrm{~ms}$ delay in the muscle activation and a $150 \mathrm{~ms}$ delay in the force at the hand. We also demonstrate that the magnitude of this reflex response is affected by the statistical properties of the visual environment in which subjects move. When the visual motor discrepancies were task-relevant, the magnitude of the reflex response was enhanced. Moreover, the response increased in a spatially localized way to the region in 
which task-relevant variability was introduced. In contrast, when faced with taskirrelevant variability, the size of the visually induced motor reflex was suppressed.

The perturbation of the hand representation in these experiments produced a motor response with similar latency to previous studies: $160 \mathrm{~ms}$ (Saunders and Knill, 2003) and 117 ms (Saunders and Knill, 2005) to changes in position and $\sim 125$ ms to changes in velocity (Brenner and Smeets, 2003). Similar latencies were also found in response to shifts of the target: $150 \mathrm{~ms}$ (Brenner and Smeets, 2003), $110 \mathrm{~ms}$ (Brenner and Smeets, 1997), 115 ms (Prablanc and Martin, 1992), and shifts of the background: $126 \mathrm{~ms}$ (Saijo et al., 2005). These latencies are generally faster than what would be associated with a voluntary response to the stimuli. By using a task, similar to one used previously (Day and Lyon, 2000), where subjects are asked to respond in the opposite direction to the normal response to the stimuli we determined the fastest possible voluntary response to the visual perturbation. The earliest distinguishable response in any of the 10 subjects occurred at $185 \mathrm{~ms}$ in the EMG and $232 \mathrm{~ms}$ in the force relative to the onset of the visual perturbation. Before this time, the early corrective responses to the visual perturbation opposing the perturbation were not suppressed. Similar responses for perturbations of the target during the reaching movement have been found (Day and Lyon, 2000), although the onset of the voluntary response to the target displacement occurred earlier than we found for the hand representation, with an average delay of $200 \mathrm{~ms}$ following the shift in target. It has been shown that subjects tend to focus their vision on the target or features of the environment coming up in the movement, rather than focusing upon the hand itself during the movement (Abrams et al., 1990; Johansson et al., 2001), which could explain the longer delay before voluntary responses were seen to shifts in the hand representation compared with shifts in the target. Overall, these results suggest that the visually induced motor response to the shift in the hand position is a fast involuntary response of the motor system acting to correct for errors in limb movements. This is the first demonstration that the fast motor responses to perturbations of the visual representation of the hand are involuntary in nature.

It is not yet clear whether this rapid motor response to the visual location of the hand is produced by the same neural circuits responsible for rapid motor responses to the target (Goodale et al., 1986; Day and Lyon, 2000), background (Saijo et al., 2005; Gomi et al., 2006) or even visual targets (Corneil et al., 2004, 2008). However, several studies have suggested that the motor response to a shift in the target is produced by a comparison of the current target location to the expected location of the hand task-irrelevant condition.
B Hand Trajectories
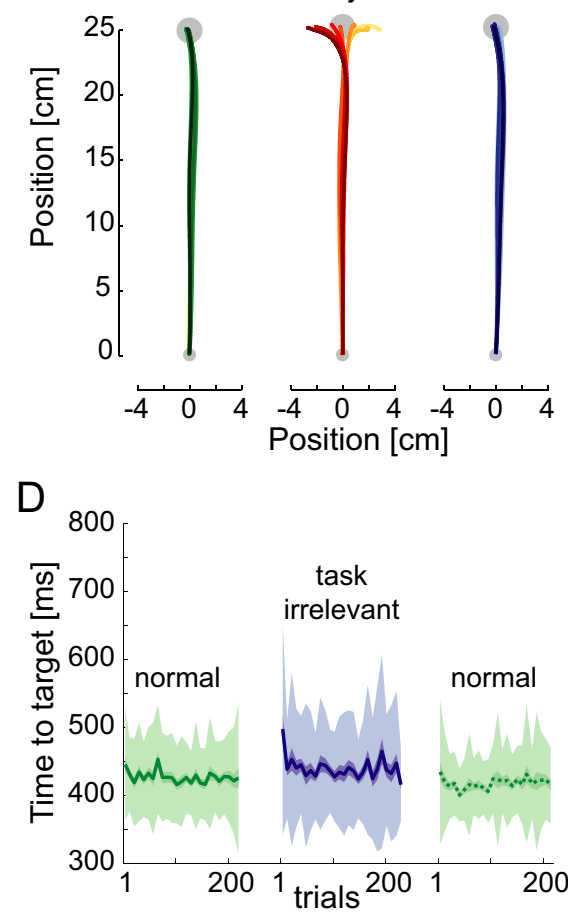

F

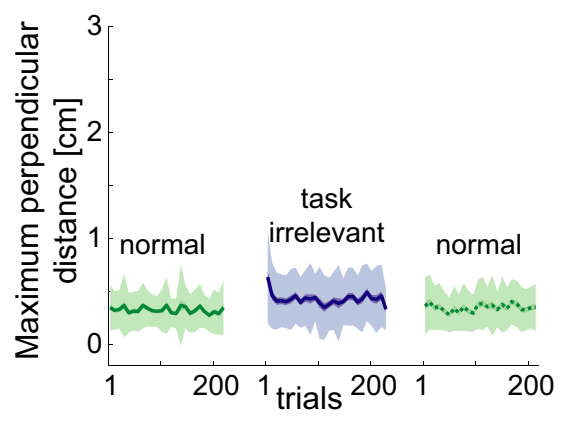

Figure 5. Kinematic measures in the visuomotor discrepancy experiment. $\boldsymbol{A}$, The mean cursor trajectories for the seven different trajectories for a single subject (normal condition: green; task-relevant condition: red; task-irrelevant condition: blue). $\boldsymbol{B}$, The mean hand trajectories for the same movements are shown for the same subject. $\boldsymbol{C}$, Time to move to the target from the onset of SEM (dark shaded region), and SD (light shaded region) are shown across all subjects for blocks of six movements. D, Time to move the target from the onset of the visual discrepancy in the task-irrelevant condition and the two normal conditions run on the isplacement was determined from the time of visual discrepancy onset. $\boldsymbol{F}$, Maximum perpendicular displacement during the

(Goodale et al., 1986; Pélisson et al., 1986; Prablanc et al., 1986; Prablanc and Martin, 1992), where the prediction would be based upon either visio-kinesthetic feedback loops, forward models of the hand based on efference copy, or combinations of these processes. If responses to the shift in target are produced by a comparison of the expected hand position with the target position, then it could be expected that the current reflex response to a shift in the visual location of the hand uses the same neural circuits.

We investigated whether the motor system controls the magnitude of this involuntary response based on the statistical properties of the environment. Previous research has shown that the CNS integrates sensory information from different sensory modalities in an optimal manner weighting each modality depending on its reliability (van Beers et al., 2002; Sober and Sabes, 2003; 
A

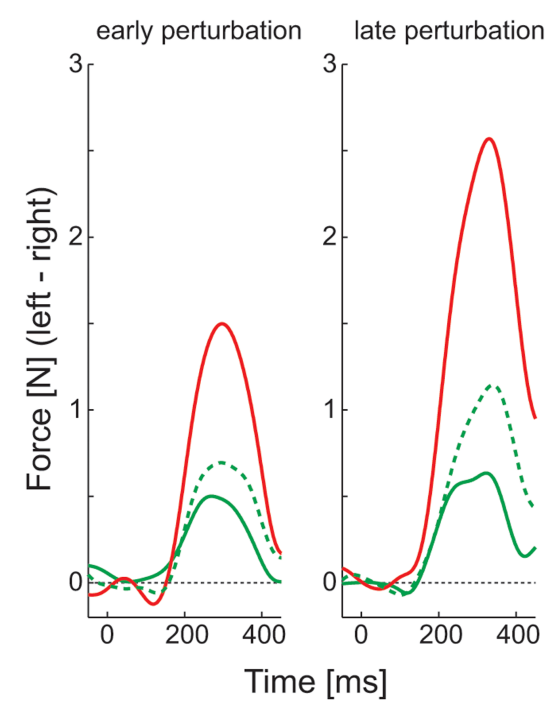

D

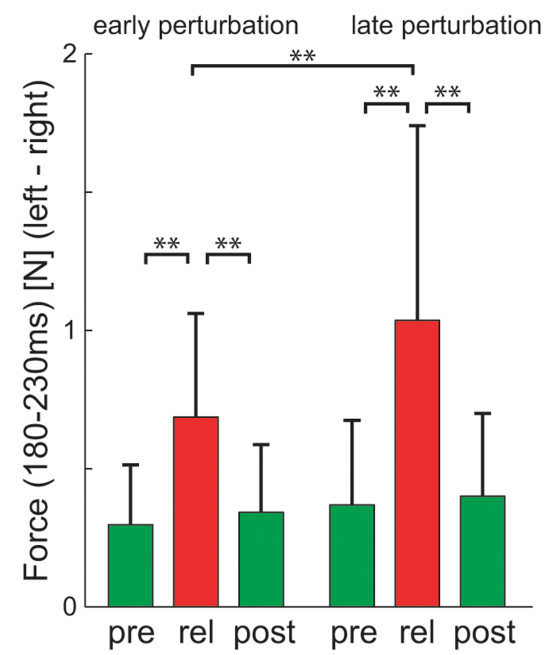

B

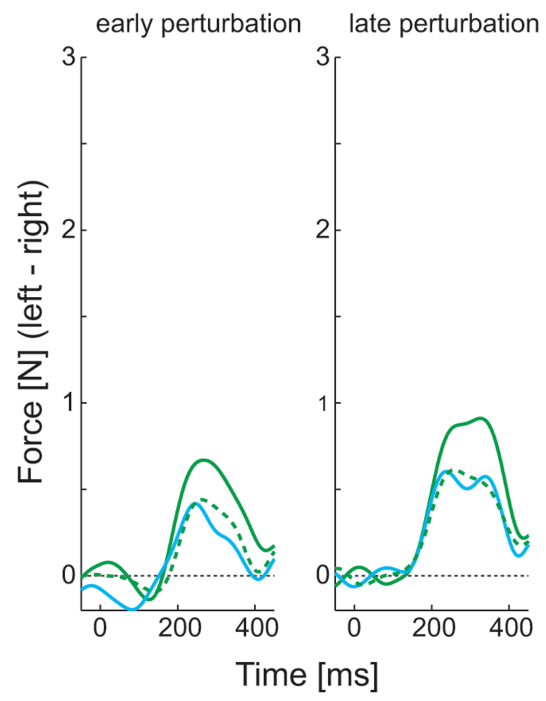

$E$

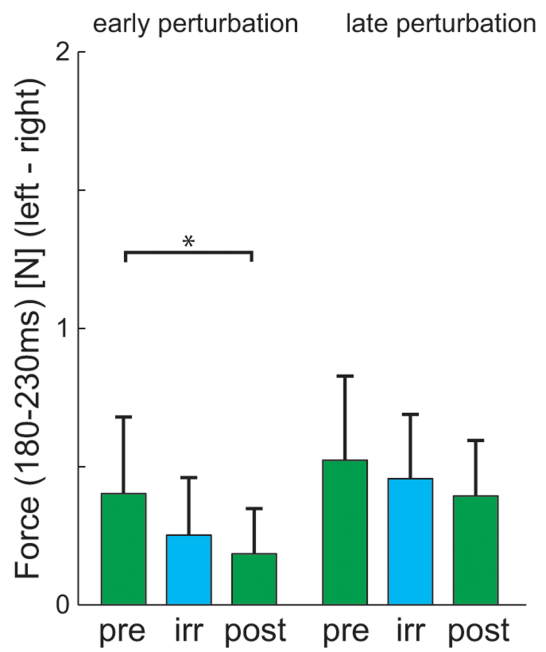

C

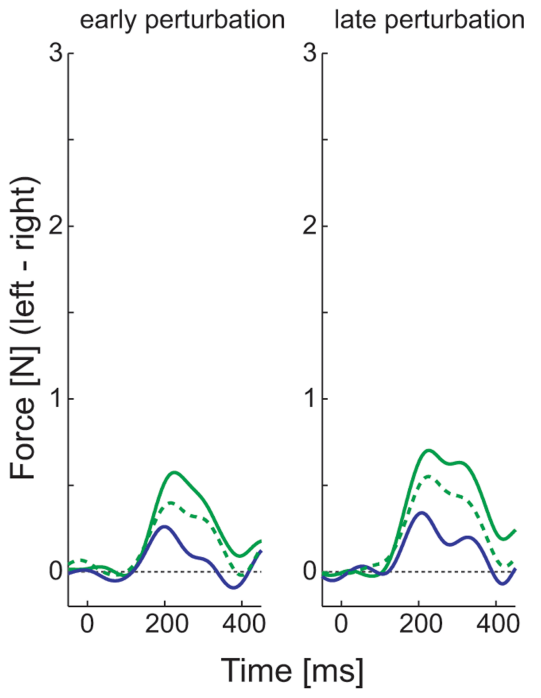

$\mathrm{F}$

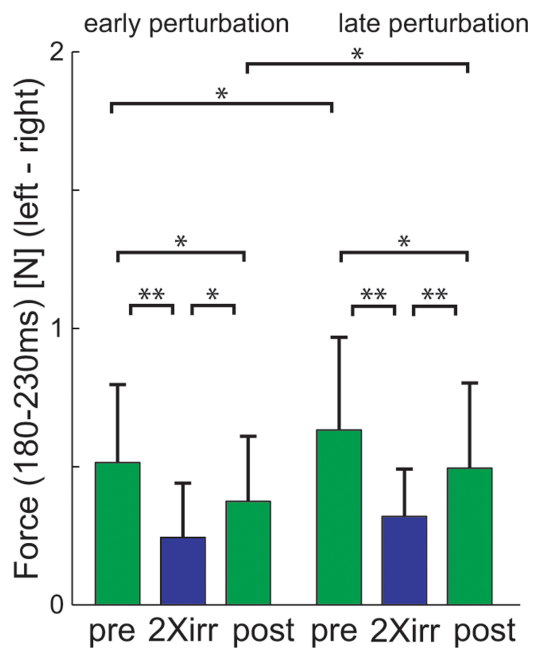

Figure 6. Steady state motor responses to the visual perturbation. $\boldsymbol{A}$, The mean force response to the early (right) and late (left) visual perturbations in the normal and task-relevant visual environments across all subjects. Responses in the first five blocks in each condition were not included. The response in the initial normal environment (pre; solid green line), task-relevant visual environment (red line), and the final normal environment (post; dotted green line) are shown as a function of time from the onset of the visual perturbation. $\boldsymbol{B}$, The mean force response to the early (right) and late (left) visual perturbations in the normal (green lines) and task-irrelevant (cyan line) visual environments. $\boldsymbol{C}$, The mean force response to the early (right) and late (left) visual perturbations in the normal (green lines) and double sized task-irrelevant (blue line) visual environments. $D$, The mean force response \pm SD over an early interval (180 -230 ms) for the task-relevant day. Statistical significant differences between the conditions were tested using Tukey's HSD post hoc test ( $\left.{ }^{*} p<0.05 ;{ }^{* *} p<0.001\right)$. $\boldsymbol{E}$, The mean force response \pm SD over an early interval $(180-230 \mathrm{~ms})$ for the task-irrelevant day. $\boldsymbol{F}$, The mean force response \pm SD over an early interval (180 - 230 ms) for the doubled sized task-irrelevant day.

Körding and Wolpert, 2004). This suggests that the CNS has some measure of the reliability of the sensory information which it is processing. In addition, optimal feedback control predicts that feedback is controlled to correct for disturbances that affect task success (Todorov and Jordan, 2002; Todorov, 2004; Liu and Todorov, 2007; Scott, 2008). We show that the reflex response under task-relevant variability exhibits a greater magnitude compared with the normal movements. Conversely under the larger task-irrelevant variability, the reflex response was decreased compared with the normal environments.

In the task-relevant condition, the reflex response to the late perturbation was significantly larger than that to the early perturbation. However, there were no differences in the response to these perturbations in the normal visual condition. This suggests that the increase for the late perturbation in the task-relevant condition does not simply reflect a nonspecific increase in the feedback gain throughout the movement but a spatially selective increase. The task-relevant visual discrepancies were applied only through the latter half of the movement, and it appears that the visuomotor reflex response was increased to a larger degree for this portion.

While the second experiment demonstrated that the reflex magnitude is adapted depending upon the task, the reflex response was still present during the first experiment where subjects were asked to respond in the same direction as the visual perturbation. However during these probe trials the lateral position of the hand was constrained by the mechanical channel. Previous work has shown that de-adaptation to force fields is slowed during these channel trials (Scheidt et al., 2000) and it appears that the mechanical channel may have also limited any adaptation of the reflex in these trials. During the second experiment where adaptation was found, the hand was free to move on 
A
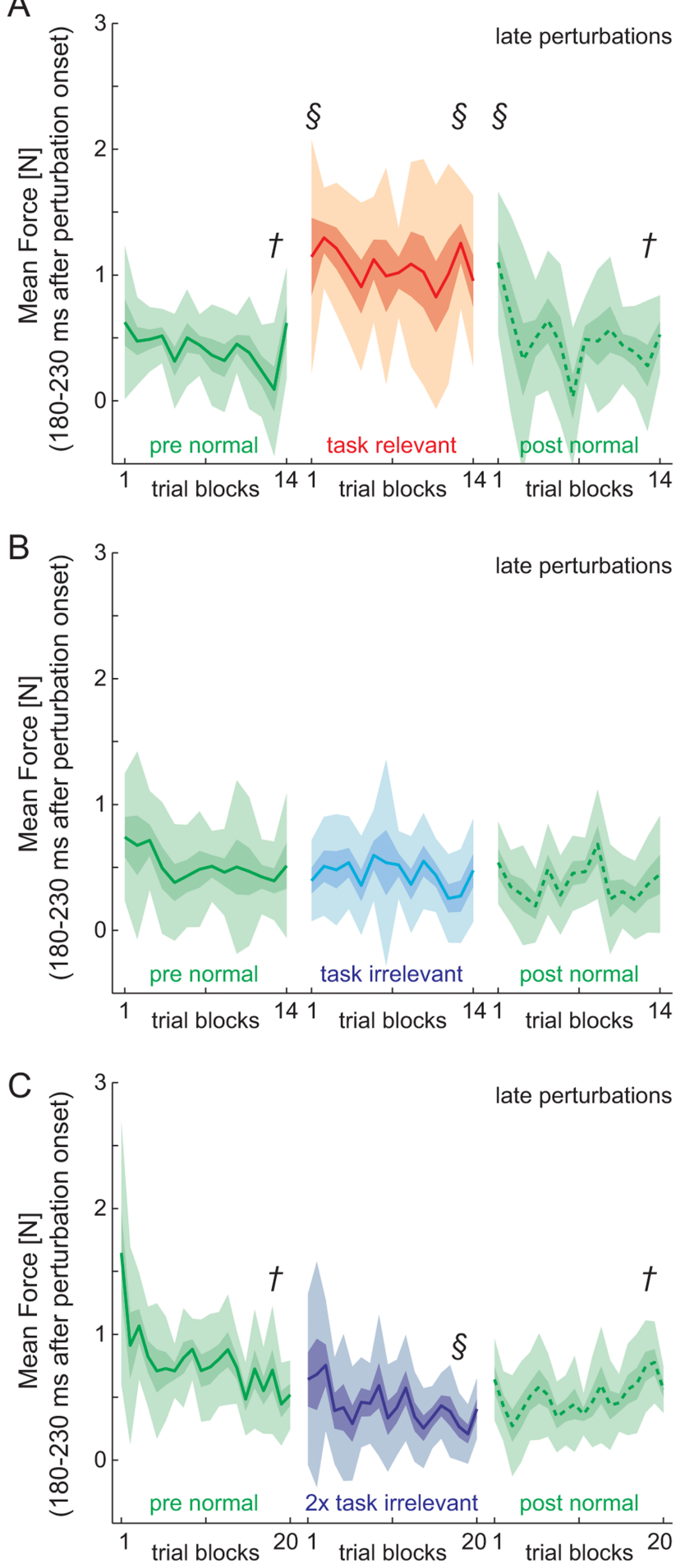

Figure 7. Time course of the adaptation to the statistical distributions. $\boldsymbol{A}$, The mean (solid line) $\pm S D$ (shaded region) force difference between right and left visual perturbations in the pre normal, task-relevant and post normal conditions across subjects. The force difference was determined for each block of 15 trials in which one of each perturbation direction was included. The mean force response was estimated over the period from 180 to $230 \mathrm{~ms}$ after the onset of the perturbation, before the timing of a voluntary response. The symbols, $\uparrow$ and $\S$, indicate membership in homogeneous groups which were significantly different from each other $(p<$ 0.05 ) as determined by the Tukey's HSD post hoc test. $\boldsymbol{B}$, The mean force difference between the right and left visual perturbations during the pre normal, task-irrelevant and post normal visual conditions. Responses have been quantified over the interval $180-230 \mathrm{~ms}$ relative to the onset of the visual perturbation. $\boldsymbol{C}$, The mean force difference between the right and left visual perturbations during the pre normal, double sized task-irrelevant and post normal visual conditions over the interval $180-230$ ms after the onset of the visual perturbation. the majority of trials so that the any corrections assisted by or errors induced by the visuomotor response could be detectable and attributable. This may indicate that the gain of the feedback response is trained by the errors in the hand position at the end of trials.

The rate of adaptation of the reflex magnitude showed a striking asymmetry. On the introduction of task-relevant variability the magnitude of the reflex response plateaued within the first block of trials. However, on the removal of task-relevant variability the change in magnitude took several blocks to return to baseline. We can interpret this asymmetry in terms of the CNS detecting changes in the environment. For example, it has been suggested that the CNS has different mechanism for determining expected and unexpected uncertainty (Yu and Dayan, 2005). There is natural variation in the perceived location of the hand due to noise in the visual and proprioceptive system and such variability is expected. However, on the introduction of our taskrelevant variability, the visual location of the hand would fall outside the normal statistical range thus reflecting unexpected uncertainty triggering an increase in reflex magnitude. Similar fast changes to variations in the statistics of the external world have previously been reported in the fly visual system (Brenner et al., 2000; Fairhall et al., 2001). However, on the removal of the discrepancies, the trials fall within the variability expected during the task-relevant discrepancy trials and therefore reflect expected uncertainty. Only over time will the CNS be able to realize that the variance of the visuomotor discrepancy has reduced thereby leading to a reduced response. Similar asymmetry has been seen in learning task distributions (Körding et al., 2004; Miyazaki et al., 2005). Therefore we expect, as we find, a quick adaptation from low to high variance conditions but a slow adaptation from high to low variance conditions.

Previous studies examining reflex responses have found that the gain of a reflex response is adapted by the CNS. However, in all previous cases the change in the reflex gain was seen either under a fixed change in the environment (Hammond, 1956; Nashner, 1976; Robinson, 1976; Miles and Eighmy, 1980; Dufresne et al., 1980; Rothwell et al., 1980; Akazawa et al., 1983; Horak and Nashner, 1986; Lacquaniti and Maioli, 1987; Llewellyn et al., 1990; De Serres and Milner, 1991; Lacquaniti et al., 1991, 1992; Doemges and Rack, 1992a,b; Johnson et al., 1993; Fitzpatrick et al., 1994; Pearson and Misiaszek, 2000; Franklin et al., 2007) or for a different state of the body (Lacquaniti and Soechting, 1984, 1986a,b; Brown and Kukulka, 1993; Zehr et al., 2003; Kurtzer et al., 2008). Our task, however, did not have a fixed change in the environment or a change in the state of the body. For both the task-relevant and task-irrelevant conditions, there were discrepancies between the actual hand position and the representation of the hand position, both with a mean of zero. However, in the task-relevant condition, the visual information is a reliable estimate of the final error whereas in the task-irrelevant condition the visual discrepancy simply increases the unreliability of the visual signal. This is the first demonstration that the CNS modulates the reflex magnitude depending on statistical properties of the environment when such changes are task relevant.

\section{References}

Abrams RA, Meyer DE, Kornblum S (1990) Eye-hand coordination: oculomotor control in rapid aimed limb movements. J Exp Psychol Hum Percept Perform 16:248-267.

Akazawa K, Milner TE, Stein RB (1983) Modulation of reflex EMG and stiffness in response to stretch of human finger muscle. J Neurophysiol 49:16-27. 
Barr CC, Schultheis LW, Robinson DA (1976) Voluntary, non-visual control of the human vestibulo-ocular reflex. Acta Otolaryngol 81:365-375.

Brenner E, Smeets JB (1997) Fast responses of the human hand to changes in target position. J Mot Behav 29:297-310.

Brenner E, Smeets JB (2003) Fast corrections of movements with a computer mouse. Spat Vis 16:365-376.

Brenner N, Bialek W, de Ruyter van Steveninck R (2000) Adaptive rescaling maximizes information transmission. Neuron 26:695-702.

Brown DA, Kukulka CG (1993) Human flexor reflex modulation during cycling. J Neurophysiol 69:1212-1224.

Capaday C, Stein RB (1986) Amplitude modulation of the soleus H-reflex in the human during walking and standing. J Neurosci 6:1308-1313.

Corneil BD, Olivier E, Munoz DP (2004) Visual responses on neck muscles reveal selective gating that prevents express saccades. Neuron 42:831-841.

Corneil BD, Munoz DP, Chapman BB, Admans T, Cushing SL (2008) Neuromuscular consequences of reflexive covert orienting. Nat Neurosci 11:13-15.

Crago PE, Houk JC, Hasan Z (1976) Regulatory actions of human stretch reflex. J Neurophysiol 39:925-935.

Day BL, Lyon IN (2000) Voluntary modification of automatic arm movements evoked by motion of a visual target. Exp Brain Res 130:159-168.

De Serres SJ, Milner TE (1991) Wrist muscle activation patterns and stiffness associated with stable and unstable mechanical loads. Exp Brain Res 86:451-458.

Doemges F, Rack PM (1992a) Task-dependent changes in the response of human wrist joints to mechanical disturbance. J Physiol 447:575-585.

Doemges F, Rack PM (1992b) Changes in the stretch reflex of the human first dorsal interosseous muscle during different tasks. J Physiol 447:563-573.

Dufresne JR, Soechting JF, Terzuolo CA (1980) Modulation of the myotatic reflex gain in man during intentional movements. Brain Res 193:67-84.

Evarts EV, Tanji J (1974) Gating of motor cortex reflexes by prior instruction. Brain Res 71:479-494.

Fairhall AL, Lewen GD, Bialek W, de Ruyter Van Steveninck RR (2001) Efficiency and ambiguity in an adaptive neural code. Nature 412:787-792.

Fitzpatrick R, Burke D, Gandevia SC (1994) Task-dependent reflex responses and movement illusions evoked by galvanic vestibular stimulation in standing humans. J Physiol 478:363-372.

Franklin DW, Liaw G, Milner TE, Osu R, Burdet E, Kawato M (2007) Endpoint stiffness of the arm is directionally tuned to instability in the environment. J Neurosci 27:7705-7716.

Gomi H, Abekawa N, Nishida S (2006) Spatiotemporal tuning of rapid interactions between visual-motion analysis and reaching movement. J Neurosci 26:5301-5308.

Goodale MA, Pelisson D, Prablanc C (1986) Large adjustments in visually guided reaching do not depend on vision of the hand or perception of target displacement. Nature 320:748-750.

Hammond PH (1956) The influence of prior instruction to the subject on an apparently involuntary neuro-muscular response. J Physiol 132:17P-18P.

Horak FB, Nashner LM (1986) Central programming of postural movements: adaptation to altered support-surface configurations. J Neurophysiol 55:1369-1381.

Johansson RS, Westling G, Bäckström A, Flanagan JR (2001) Eye-hand coordination in object manipulation. J Neurosci 21:6917-6932.

Johnson MT, Kipnis AN, Lee MC, Ebner TJ (1993) Independent control of reflex and volitional EMG modulation during sinusoidal pursuit tracking in humans. Exp Brain Res 96:347-362.

Körding KP, Wolpert DM (2004) Bayesian integration in sensorimotor learning. Nature 427:244-247.

Körding KP, Ku SP, Wolpert DM (2004) Bayesian integration in force estimation. J Neurophysiol 92:3161-3165.

Kurtzer IL, Pruszynski JA, Scott SH (2008) Long-latency reflexes of the human arm reflect an internal model of limb dynamics. Curr Biol 18:449-453.

Lacquaniti F, Maioli C (1987) Anticipatory and reflex coactivation of antagonist muscles in catching. Brain Res 406:373-378.

Lacquaniti F, Soechting JF (1984) Behav of the stretch reflex in a multijointed limb. Brain Res 311:161-166.

Lacquaniti F, Soechting JF (1986a) EMG responses to load perturbations of the upper limb: effect of dynamic coupling between shoulder and elbow motion. Exp Brain Res 61:482-496.

Lacquaniti F, Soechting JF (1986b) Responses of mono- and bi-articular muscles to load perturbations of the human arm. Exp Brain Res 65:135-144.

Lacquaniti F, Borghese NA, Carrozzo M (1991) Transient reversal of the stretch reflex in human arm muscles. J Neurophysiol 66:939-954.
Lacquaniti F, Borghese NA, Carrozzo M (1992) Internal models of limb geometry in the control of hand compliance. J Neurosci 12:1750-1762.

Liu D, Todorov E (2007) Evidence for the flexible sensorimotor strategies predicted by optimal feedback control. J Neurosci 27:9354-9368.

Llewellyn M, Yang JF, Prochazka A (1990) Human H-reflexes are smaller in difficult beam walking than in normal treadmill walking. Exp Brain Res 83:22-28.

Miles FA, Eighmy BB (1980) Long-term adaptive changes in primate vestibuloocular reflex. I. Behavioral observations. J Neurophysiol 43:1406-1425.

Miyazaki M, Nozaki D, Nakajima Y (2005) Testing Bayesian models of human coincidence timing. J Neurophysiol 94:395-399.

Nashner LM (1976) Adapting reflexes controlling the human posture. Exp Brain Res 26:59-72.

Oldfield RC (1971) The assessment and analysis of handedness: the Edinburgh inventory. Neuropsychologia 9:97-113.

Pearson KG, Misiaszek JE (2000) Use-dependent gain change in the reflex contribution to extensor activity in walking cats. Brain Res 883:131-134.

Pélisson D, Prablanc C, Goodale MA, Jeannerod M (1986) Visual control of reaching movements without vision of the limb. II. Evidence of fast unconscious processes correcting the trajectory of the hand to the final position of a double-step stimulus. Exp Brain Res 62:303-311.

Prablanc C, Martin O (1992) Automatic control during hand reaching at undetected two-dimensional target displacements. J Neurophysiol 67:455-469.

Prablanc C, Pélisson D, Goodale MA (1986) Visual control of reaching movements without vision of the limb. I. Role of retinal feedback of target position in guiding the hand. Exp Brain Res 62:293-302.

Pruszynski JA, Kurtzer I, Scott SH (2008) Rapid motor responses are appropriately tuned to the metrics of a visuospatial task. J Neurophysiol 100:224-238.

Robinson DA (1976) Adaptive gain control of vestibuloocular reflex by the cerebellum. J Neurophysiol 39:954-969.

Rothwell JC, Traub MM, Marsden CD (1980) Influence of voluntary intent on the human long-latency stretch reflex. Nature 286:496-498.

Saijo N, Murakami I, Nishida S, Gomi H (2005) Large-field visual motion directly induces an involuntary rapid manual following response. J Neurosci 25:4941-4951.

Sarlegna F, Blouin J, Bresciani JP, Bourdin C, Vercher JL, Gauthier GM (2003) Target and hand position information in the online control of goal-directed arm movements. Exp Brain Res 151:524-535.

Sarlegna F, Blouin J, Vercher JL, Bresciani JP, Bourdin C, Gauthier GM (2004) Online control of the direction of rapid reaching movements. Exp Brain Res 157:468-471.

Saunders JA, Knill DC (2003) Humans use continuous visual feedback from the hand to control fast reaching movements. Exp Brain Res 152:341-352.

Saunders JA, Knill DC (2004) Visual feedback control of hand movements. J Neurosci 24:3223-3234.

Saunders JA, Knill DC (2005) Humans use continuous visual feedback from the hand to control both the direction and distance of pointing movements. Exp Brain Res 162:458-473.

Scheidt RA, Reinkensmeyer DJ, Conditt MA, Rymer WZ, Mussa-Ivaldi FA (2000) Persistence of motor adaptation during constrained, multi-joint, arm movements. J Neurophysiol 84:853-862.

Scott SH (2008) Inconvenient truths about neural processing in primary motor cortex. J Physiol 586:1217-1224.

Sober SJ, Sabes PN (2003) Multisensory integration during motor planning. J Neurosci 23:6982-6992.

Soechting JF, Lacquaniti F (1983) Modification of trajectory of a pointing movement in response to a change in target location. J Neurophysiol 49:548-564.

Todorov E (2004) Optimality principles in sensorimotor control. Nat Neurosci 7:907-915.

Todorov E, Jordan MI (2002) Optimal feedback control as a theory of motor coordination. Nat Neurosci 5:1226-1235.

van Beers RJ, Wolpert DM, Haggard P (2002) When feeling is more important than seeing in sensorimotor adaptation. Curr Biol 12:834-837.

Yu AJ, Dayan P (2005) Uncertainty, neuromodulation, and attention. Neuron 46:681-692.

Zehr EP, Collins DF, Frigon A, Hoogenboom N (2003) Neural control of rhythmic human arm movement: phase dependence and task modulation of hoffmann reflexes in forearm muscles. J Neurophysiol 89: $12-21$. 